\title{
Exact and Explicit Solutions of Whitham-Broer-Kaup Equations in Shallow Water
}

\author{
Baodan Tian, Yanhong Qiu \\ School of science, Southwest University of Science and Technology, Mianyang, China \\ Email address: \\ tianbaodan@swust.edu.cn (Baodan Tian), qiuyanhong@swust.edu.cn (Yanhong Qiu)
}

To cite this article:

Baodan Tian, Yanhong Qiu. Exact and Explicit Solutions of Whitham-Broer-Kaup Equations in Shallow Water. Pure and Applied Mathematics Journal. Vol. 5, No. 6, 2016, pp. 174-180. doi: 10.11648/j.pamj.20160506.11

Received: September 2, 2016; Accepted: September 18, 2016; Published: October 17, 2016

\begin{abstract}
In this paper, a simple direct method is presented to find equivalence transformation of a nonlinear WhithamBroer-Kaup equations. Applying this equivalence transformation, we can obtain the symmetry group theorem of the Whitham-Broer-Kaup equations and then derive series of new exact and explicit solutions of the Whitham-Broer-Kaup equations according to solutions of the previous references.
\end{abstract}

Keywords: Whitham-Broer-Kaup Equations, Direct Method, Equivalence Transformation, Symmetry, Explicit Solutions

\section{Introduction}

Since the discovery of the soliton in 1965 by Zabusky and Kruskal, a large class of nonlinear evolution equations (NLEEs) have been derived and widely applied in various branches of physics and applied mathematics like condensed matter, nonlinear optics, fluid mechanics, plasma physics, theory of turbulence, ocean dynamics, biophysics and star formation. On the other hand, to better understand the nonlinear mechanisms in different physical contexts, many authors have diligently applied themselves to finding the exact analytical solutions for these NLEEs including the soliton solutions, periodic solutions and rational solutions.

In the present paper, we would like to consider the coupled Whitham-Broer-Kaup (WBK) equations which have been studied by Whitham [16], Broer [2] and Kaup [10]. The WBK equations are as follows,

$$
\left\{\begin{array}{l}
u_{t}+u u_{x}+v_{x}+\beta u_{x x}=0, \\
v_{t}+(u v)_{x}-\beta v_{x x}+\alpha u_{x x x}=0
\end{array}\right.
$$

The WBK equations describe the propagation of shallow water waves, with different dispersion relations. Where $u=u(x, t)$ is the horizontal velocity, $v=v(x, t)$ is the height that deviates from equilibrium position of the liquid, and $\alpha, \beta$ are constants which are represented in different diffusion powers. WBK equations are very good models to describe dispersive wave. For the background materials of model equation, we refer to the paper $[2,10,16]$.

If $\alpha=0$ and $\beta \neq 0$, Eq.(1) is classic long wave equations that describe shallow water wave with diffusion [17]. If $\alpha=1$ and $\beta=0$, Eq.(1) is modified Boussinesq equations [8]. Kaup [10] and Ablowitz [1] studied inverse transformation solution for the special case of Eq.(1), Kupershmidt discussed their symmetries and conservation laws. By using of Backlund transformation, Fan [8] found three pairs of solutions of WBK equation. Xie et al. [17] made use of hyperbolic function method and $\mathrm{Wu}$ elimination method obtained four pairs of solutions of WBK equation. Chen et al. [3] present a more general transform ation and applied it to WBK equation and obtained some traveling wave solutions. $\mathrm{Xu}$ et al. [20] obtained abundant solitary- wave solutions and reveal some novel solitary-wave structures under certain parametric conditions for the WBK equation by tanh-function method. $\mathrm{Xu}$ [18] presented an elliptic equation method for constructing new types of elliptic function solutions for the WBK equation. Sirendaoreji [15] suggested a new auxiliary ordinary differential equation method and constructed exact travelling wave solutions of the WBK equation in a unified way and so on.

However, there are still many researchers are discussing the exact and explicit solutions for this WBK equation $[4,5$, $7,9,14,19,21]$ by many new methods, and all kinds of solutions, such as traveling wave solutions, tanh-function solutions, elliptic function solutions, Jacobi elliptic function 
solutions, rational function solutions, exponential function solutions of the WBK equation are obtained in this references.

Naturally, an interesting questions is: Can we find a simple method to obtain a transformation between the present solutions and the new ones? If we find this transformation, we can obtain more and more all kinds of new solutions by the present references. As we know, one of the way to realize this aim is backland method of transformation and method of Lie-point symmetry group [13].

On the other hand, Clarkson and Kruskal [6] introduced a simple direct method to derive symmetry reductions of a nonlinear system without using any group theory. For many types of nonlinear systems, the method can be used to find all the possible similarity reductions. Recently, Lou and Ma [11, 12] generalized a new simple direct method basing on CK's method, which is much simpler than the traditional Lie-point symmetry group.

Thus, in this paper, motivated by these ideas, the authors would like to obtain some new exact and explicit solutions by the simple direct method. And the organization of the paper is as follow. In the next section, we obtain the equivalence transformation of the WBK equation by the direct method. In section 3, we obtain series of new solutions of the WBK equation basing on the present references.

$$
\begin{array}{r}
\beta^{3} n(x, t) \tau_{x}^{2} \cdot U_{\xi \xi \xi \xi}+\beta q(x, t) \tau_{x} \cdot V_{\xi \xi}+F\left(x, t, U, V, U_{\xi}, V_{\xi}, \cdots\right)=0, \\
-\alpha \beta^{3} n(x, t) \tau_{x}^{3} \cdot U_{\xi \xi \xi \xi \xi \xi}-\beta^{3} q(x, t) \tau_{x}^{2} \cdot V_{\xi \xi \xi \xi}+G\left(x, t, U, V, U_{\xi}, V_{\xi}, \cdots\right)=0,
\end{array}
$$

where the function $F\left(x, t, U, V, U_{\xi}, V_{\xi}, \cdots\right)$ is independent of $U_{\xi \xi \xi \xi}$ and $V_{\xi \xi}$, while the function $G\left(x, t, U, V, U \xi, V_{\xi}, \cdots\right)$ is independent of $U_{\xi \xi \xi \xi \xi \xi}$ and $V_{\xi \xi \xi \xi}$. So it is necessary to take the coefficient of $U_{\xi \xi \xi \xi}$ and $V_{\xi \xi}$ in Eq.(4), $U_{\xi \xi \xi \xi \xi \xi}$ and $V_{\xi \xi \xi \xi}$ in Eq.(5) being zero.

which leads to,

$$
-\beta^{3} n(x, t) \tau_{x}^{2}=0, \beta q(x, t) \tau_{x}=0, \alpha \beta^{3} n(x, t) \tau_{x}^{3}=0, \beta^{3} q(x, t) \tau_{x}^{2}=0
$$

Then we can get $\tau_{x}=0$, that is, $\tau$ is dependent of the variable $x$, so

$$
\tau=\tau(t)
$$

At this time, if we substitute Eq.(7) into Eq.(4) and (5), then these two equations are reduced to the following

$$
\begin{array}{r}
\beta n\left[\xi_{x}^{2}-\tau^{\prime}(t)\right] \cdot U_{\xi \xi}+n\left[n \xi_{x}-\tau^{\prime}(t)\right] U U_{\xi}+n n_{x} U^{2}+F_{1}\left(x, t, U, V, U_{\xi}, V_{\xi}\right)=0 \\
\alpha\left[n(x, t) \xi_{x}^{3}-q(x, t) \tau^{\prime}(t)\right] \cdot U_{\xi \xi \xi}+\beta q(x, t)\left[\tau^{\prime}(t)-\xi_{x}^{2}\right] \cdot V_{\xi \xi}+G_{1}\left(x, t, U, V, U_{\xi}, V_{\xi}, \cdots\right)=0
\end{array}
$$

where the function $F_{1}\left(x, t, U, V, U_{\xi}, V_{\xi}\right)$ is independent of $U_{\xi \xi}, U U_{\xi}$ and $U^{2}$, while the function $G_{1}\left(x, t, U, V, U_{\xi}, V_{\xi}, \cdots\right)$ is independent of $U_{\xi \xi \xi}$ and $V_{\xi \xi}$.

In the same way, it is necessary to take the coefficients of $U_{\xi \xi}, U U_{\xi}$ and $U^{2}$ in Eq.(8), $U_{\xi \xi \xi}$ and $V_{\xi \xi}$ in Eq.(9) being zero. That is,

$$
\begin{gathered}
\beta n\left[\xi_{x}^{2}-\tau^{\prime}(t)\right]=n\left[n \xi_{x}-\tau^{\prime}(t)\right]=n n_{x}=0, \\
\alpha\left[n(x, t) \xi_{x}^{3}-q(x, t) \tau^{\prime}(t)\right]=\beta q(x, t)\left[\tau^{\prime}(t)-\xi_{x}^{2}\right]=0,
\end{gathered}
$$


Thus, it is easy to obtain

$$
\tau^{\prime}(t)=\xi_{x}^{2}, n(x, t)=n(t)=\xi_{x}(x, t), q(x, t)=q(t)=n^{2}(t),
$$

Therefore, if we substitute Eq.(12) into Eq.(8) and (9), then these two equations can be reduced to the following

$$
\begin{gathered}
n\left[\xi_{t}+m \xi_{x}\right] \cdot U_{\xi}+\left[n^{\prime}(t)+n m_{x}\right] U+\left[m_{t}+m m_{x}+p_{x}+\beta m_{x x}\right]=0 \\
{\left[n \xi_{x}-q \tau^{\prime}(t)\right] \cdot U V_{\xi}+n p \xi_{x} \cdot U_{\xi}+\left[\xi_{t}+m \xi_{x}\right] V_{\xi}+n p_{x} \cdot U+\left[q^{\prime}(t)+q m_{x}\right] \cdot V} \\
+\left[p_{t}+m p_{x}+p m_{x}-\beta p_{x x}+\alpha m_{x x x}\right]=0
\end{gathered}
$$

Also, it is necessary to take all the coefficients of the Eq.(13) and Eq.(14), which leads to

$$
\begin{aligned}
\xi_{t}+m \xi_{x}=0 & , n^{\prime}(t)+n m_{x}=0, m_{t}+m m_{x}+p_{x}+\beta m_{x x}=0 . \\
& \left\{\begin{array}{l}
n \xi_{x}-q \tau^{\prime}(t)=0 \\
n p \xi_{x}=0 \\
n p_{x}=0 \\
q^{\prime}(t)+q m_{x}=0 \\
p_{t}+m p_{x}+p m_{x}-\beta p_{x x}+\alpha m_{x x x}=0
\end{array}\right.
\end{aligned}
$$

From Eq.(12), Eq.(15) and Eq.(16) one can get

$$
p(x, t)=0, q(x, t)=1, m(x, t)=c_{1}, n(x, t)=\delta, \xi(x, t)=\delta x-c_{1} \delta t+c_{2}, \tau(x, t)=t+c_{3}
$$

where $c_{1}, c_{2}, c_{3}$ are arbitrary constants and $\delta= \pm 1$.

Thus, we can easily get the following symmetry group theorem.

Theorem 1. If $U=U(x, t)$ and $V=V(x, t)$ is a given solution of the WBK equations, then

$$
\begin{gathered}
u(x, t)=c_{1}+\delta U\left(\delta x-c_{1} \delta t+c_{2}, t+c_{3}\right) \\
v(x, t)=V\left(\delta x-c_{1} \delta t+c_{2}, t+c_{3}\right)
\end{gathered}
$$

is also a solutions of the WBK equations.

According to the formula (18) and (19), we obtain the relationship between the new explicit solutions and the old ones of the WBK equations, which is the so-called equivalence transformation of the Eq.(1). Also it is obvious to see that (18) and (19) is an auto-backlund transformation of the WBK equations (1).

\section{Some New Exact and Explicit Solutions of the WBK Equations}

Utilizing the conclusion of the Theorem 1, we can obtain following exact and explicit solutions of the WBK equations in this section.

Family 1. From reference [4] we know that WBK equations have the following solution:

$$
\begin{gathered}
U(\xi, \tau)=a_{0}+\frac{a_{1}\left(\mu_{1}\left(\tanh (\zeta) \pm i \sec h(\zeta)+\mu_{2}(\sec (\zeta) \pm \tan (\zeta))\right)\right.}{\mu_{1}\left(\mu_{1}(\tanh (\zeta) \pm i \sec h(\zeta))+\mu_{2}(\sec (\zeta) \pm \tan (\zeta))+1\right)} \\
V(\xi, \tau)=A_{0}+\frac{a_{1}\left( \pm \beta k \mu_{2}-\beta k \mu_{1}-\lambda-a_{0}\right)\left(\mu_{1}(\tanh (\zeta) \pm i \sec h(\zeta))+\mu_{2}(\sec (\zeta) \pm \tan (\zeta))\right)}{\mu_{1}\left(\mu_{1}(\tanh (\zeta) \pm i \sec h(\zeta))+\mu_{2}(\sec (\zeta) \pm \tan (\zeta))+1\right)} \\
-\frac{a_{1}\left(\beta k \pm \beta k \mu_{1} \mu_{2}-\mu_{1}^{2} \beta k+a_{1}\right)(\tanh (\zeta) \pm i \sec h(\zeta))^{2}}{2\left(\mu_{1}(\tanh (\zeta) \pm i \sec h(\zeta))+\mu_{2}(\sec (\zeta) \pm \tan (\zeta))+1\right)^{2}} \\
-\frac{a_{1} \mu_{2}\left( \pm \beta k \mu_{1} \mu_{2}-\mu_{1}^{2} \beta k+a_{1}\right)(\tanh (\zeta) \pm i \sec h(\zeta))(\sec (\zeta) \pm \tan (\zeta))}{\mu_{1}\left(\mu_{1}(\tanh (\zeta) \pm i \sec h(\zeta))+\mu_{2}(\sec (\zeta) \pm \tan (\zeta))+1\right)^{2}} \\
-\frac{a_{1} \mu_{2}\left( \pm \beta k \mu_{1} \mu_{2} \pm \mu_{1} \mu_{2}^{2} \beta k-\mu_{1}^{2} \mu_{2} \beta k+a_{1} \mu_{2}\right)(\sec (\zeta) \pm \tan (\zeta))^{2}}{2 \mu_{1}^{2}\left(\mu_{1}(\tanh (\zeta) \pm i \sec h(\zeta))+\mu_{2}(\sec (\zeta) \pm \tan (\zeta))+1\right)^{2}}
\end{gathered}
$$

where $\zeta=k(\xi+\lambda \tau), a_{0}, A_{0}, a_{1}, \mu_{1}, \mu_{2}, k$ and $\lambda$ are arbitrary constants. 
Applying the equivalence transformation (18) and (19) we obtain following combining tanh, sech, sec and tan function solutions of the WBK equations:

$$
\begin{gathered}
u_{1}(x, t)=a_{0}+\frac{a_{1}\left(\mu_{1}\left(\tanh (\eta) \pm i \sec h(\eta)+\mu_{2}(\sec (\eta) \pm \tan (\eta))\right)\right.}{\mu_{1}\left(\mu_{1}(\tanh (\eta) \pm i \sec h(\eta))+\mu_{2}(\sec (\eta) \pm \tan (\eta))+1\right)} \\
v_{1}(x, t)=A_{0}+\frac{a_{1}\left( \pm \beta k \mu_{2}-\beta k \mu_{1}-\lambda-a_{0}\right)\left(\mu_{1}(\tanh (\eta) \pm i \sec h(\eta))+\mu_{2}(\sec (\eta) \pm \tan (\eta))\right)}{\mu_{1}\left(\mu_{1}(\tanh (\eta) \pm i \sec h(\eta))+\mu_{2}(\sec (\eta) \pm \tan (\eta))+1\right)} \\
-\frac{a_{1}\left(\beta k \pm \beta k \mu_{1} \mu_{2}-\mu_{1}^{2} \beta k+a_{1}\right)(\tanh (\eta) \pm i \sec h(\eta))^{2}}{2\left(\mu_{1}(\tanh (\eta) \pm i \sec h(\eta))+\mu_{2}(\sec (\eta) \pm \tan (\eta))+1\right)^{2}} \\
-\frac{a_{1} \mu_{2}\left( \pm \beta k \mu_{1} \mu_{2}-\mu_{1}^{2} \beta k+a_{1}\right)(\tanh (\eta) \pm i \sec h(\eta))(\sec (\eta) \pm \tan (\eta))}{\mu_{1}\left(\mu_{1}(\tanh (\eta) \pm i \sec h(\eta))+\mu_{2}(\sec (\eta) \pm \tan (\eta))+1\right)^{2}} \\
-\frac{a_{1} \mu_{2}\left( \pm \beta k \mu_{1} \mu_{2} \pm \mu_{1} \mu_{2}^{2} \beta k-\mu_{1}^{2} \mu_{2} \beta k+a_{1} \mu_{2}\right)(\sec (\zeta) \pm \tan (\zeta))^{2}}{2 \mu_{1}^{2}\left(\mu_{1}(\tanh (\zeta) \pm i \sec h(\zeta))+\mu_{2}(\sec (\zeta) \pm \tan (\zeta))+1\right)^{2}}
\end{gathered}
$$

where $\eta=k\left(\delta x-c_{1} \delta t+c_{2}\right)+k \lambda\left(t+c_{3}\right)=k \delta x+k\left(\lambda-c_{1} \delta\right) t+k\left(c_{2}+\lambda c_{3}\right), a_{0}, A_{0}, a_{1}, \mu_{1}, \mu_{2}, k$ and $\lambda$ are arbitrary constants, $\delta= \pm 1$.

Family 2. From reference [9] we know that WBK equations have the following solutions:

$$
\begin{gathered}
U(\xi, \tau)=a_{0}+b_{1} \sqrt{\frac{d_{2}}{d_{0}}} \operatorname{csch}\left(\sqrt{d_{2}} \zeta\right)+k_{1} \sqrt{d_{2}} \operatorname{coth}\left(\sqrt{d_{2}} \zeta\right), \\
V(\xi, \tau)=A_{0}+B_{1} \sqrt{\frac{d_{2}}{d_{0}}} \operatorname{csch}\left(\sqrt{d_{2}} \zeta\right)+\frac{B_{2} d_{2}}{d_{0}} \operatorname{csch}\left(\sqrt{d_{2}} \zeta\right)+\frac{P_{1}}{\sqrt{d_{0}}} \sinh \left(\sqrt{d_{2}} \zeta\right) \\
+\frac{K_{2} d_{2}}{\sqrt{d_{0}}} \operatorname{coth}\left(\sqrt{d_{2}} \zeta\right) \operatorname{csch}\left(\sqrt{d_{2}} \zeta\right)+\frac{P_{2}}{\sqrt{d_{0}}} \tanh \left(\sqrt{d_{2}} \zeta\right) .
\end{gathered}
$$

where $\zeta=k(\xi+\lambda \tau), \lambda, k_{1}, K_{2}, A_{0}, B_{1}, B_{2}, d_{0}, d_{2}$ and $P_{2}$ are mentioned in Eq.(3.5) in [9]. $a_{0}, b_{1}$ and $P_{1}$ are arbitrary constants.

So we obtain following hyperbolic function solutions of the WBK equations:

$$
\begin{gathered}
u_{2}(x, t)=a_{0}+b_{1} \sqrt{\frac{d_{2}}{d_{0}}} \operatorname{csch}\left(\sqrt{d_{2}} \eta\right)+k_{1} \sqrt{d_{2}} \operatorname{coth}\left(\sqrt{d_{2}} \eta\right), \\
v_{2}(x, t)=A_{0}+B_{1} \sqrt{\frac{d_{2}}{d_{0}}} \operatorname{csch}\left(\sqrt{d_{2}} \eta\right)+\frac{B_{2} d_{2}}{d_{0}} \operatorname{csch}\left(\sqrt{d_{2}} \eta\right)+\frac{P_{1}}{\sqrt{d_{0}}} \sinh \left(\sqrt{d_{2}} \eta\right) \\
+\frac{K_{2} d_{2}}{\sqrt{d_{0}}} \operatorname{coth}\left(\sqrt{d_{2}} \eta\right) \operatorname{csch}\left(\sqrt{d_{2}} \eta\right)+\frac{P_{2}}{\sqrt{d_{0}}} \tanh \left(\sqrt{d_{2}} \eta\right) .
\end{gathered}
$$

where $\eta=k\left(\delta x-c_{1} \delta t+c_{2}\right)+k \lambda\left(t+c_{3}\right)=k \delta x+k\left(\lambda-c_{1} \delta\right) t+k\left(c_{2}+\lambda c_{3}\right), \lambda, k_{1}, K_{2}, A_{0}, B_{1}, B_{2}, d_{0}, d_{2}$ and $P_{2}$ are mentioned in Eq.(3.5) in [9], $\delta= \pm 1$.

Family 3. From reference [18] we know that WBK equations have the following solutions:

$$
U(\xi, \tau)=c \pm k \sqrt{A\left(\alpha+\beta^{2}\right)}\left[\frac{\alpha_{1}+\alpha_{2}+\alpha_{3}}{2}-\frac{\alpha_{1} \alpha_{2} s n^{2}(B \zeta, m)}{\alpha_{1}-\alpha_{2} c n^{2}(B \zeta, m)}\right]
$$




$$
\begin{aligned}
V(\xi, \tau) & =\frac{k^{2} A\left(\alpha+\beta^{2}\right)\left(\alpha_{1}^{2}+\alpha_{2}^{2}+\alpha_{3}^{2}-2 \alpha_{1} \alpha_{2}-2 \alpha_{1} \alpha_{3}-2 \alpha_{2} \alpha_{3}\right)}{4} \\
& +\frac{k^{2} A \alpha_{1} \alpha_{2}\left(\alpha+\beta^{2}\right)\left(\alpha_{1}+\alpha_{2}+\alpha_{3}\right) s n^{2}(B \zeta, m)}{\alpha_{1}-\alpha_{2} c n^{2}(B \zeta, m)}-\frac{2 k^{2} A \alpha_{1}^{2} \alpha_{2}^{2}\left(\alpha+\beta^{2}\right) s n^{4}(B \zeta, m)}{\left(\alpha_{1}-\alpha_{2} c n^{2}(B \zeta, m)\right)^{2}} \\
& \mp \frac{4 k^{2} \beta^{2} \alpha_{1} \alpha_{2}\left(\alpha_{1}-\alpha_{2}\right) \sqrt{A\left(\alpha+\beta^{2}\right)} \operatorname{sn}(B \zeta, m) \operatorname{cn}(B \zeta, m) d n(B \zeta, m)}{\left(\alpha_{1}-\alpha_{2} c n^{2}(B \zeta, m)\right)^{2}} .
\end{aligned}
$$

where $\zeta=k\left(\xi-c \tau+\xi_{0}\right), k, c$ are arbitrary constants and $B, m$ refers to formula (4) in [18].

So we obtain following elliptic function solutions of the WBK equations:

$$
\begin{gathered}
u_{3}(x, t)=c \pm k \sqrt{A\left(\alpha+\beta^{2}\right)}\left[\frac{\alpha_{1}+\alpha_{2}+\alpha_{3}}{2}-\frac{\alpha_{1} \alpha_{2} s n^{2}(B \eta, m)}{\alpha_{1}-\alpha_{2} c n^{2}(B \eta, m)}\right], \\
v_{4}(x, t)=\frac{k^{2} A\left(\alpha+\beta^{2}\right)\left(\alpha_{1}^{2}+\alpha_{2}^{2}+\alpha_{3}^{2}-2 \alpha_{1} \alpha_{2}-2 \alpha_{1} \alpha_{3}-2 \alpha_{2} \alpha_{3}\right)}{4} \\
+\frac{k^{2} A \alpha_{1} \alpha_{2}\left(\alpha+\beta^{2}\right)\left(\alpha_{1}+\alpha_{2}+\alpha_{3}\right) s n^{2}(B \eta, m)}{\alpha_{1}-\alpha_{2} c n^{2}(B \eta, m)}-\frac{2 k^{2} A \alpha_{1}^{2} \alpha_{2}^{2}\left(\alpha+\beta^{2}\right) s n^{4}(B \eta, m)}{\left(\alpha_{1}-\alpha_{2} c n^{2}(B \eta, m)\right)^{2}} \\
\mp \frac{4 k^{2} \beta^{2} \alpha_{1} \alpha_{2}\left(\alpha_{1}-\alpha_{2}\right) \sqrt{A\left(\alpha+\beta^{2}\right)} \operatorname{sn}(B \eta, m) \operatorname{cn}(B \eta, m) d n(B \eta, m)}{\left(\alpha_{1}-\alpha_{2} c n^{2}(B \eta, m)\right)^{2}} .
\end{gathered}
$$

where $\eta=k\left(\delta x-c_{1} \delta t+c_{2}\right)-c k\left(t+c_{3}\right)+k \xi_{0}=k \delta x-k\left(c+c_{1} \delta\right) t+k\left(c_{2}-c c_{3}+\xi_{0}\right), k, c$ are arbitrary constants and $B, m$ refers to formula (4) in [18], $\delta= \pm 1$.

Family 4. From reference [18] we know that WBK equations have the following solutions:

$$
\begin{gathered}
U_{1}(\xi, \tau)= \pm R \sqrt{\alpha+\beta^{2}}(\tan \zeta+\sec \zeta)-\lambda, \\
V_{1}(\xi, \tau)=\left[-R^{2}\left(\alpha+\beta^{2}\right)+R^{2} \beta \sqrt{\alpha+\beta^{2}}\right]\left(\tan ^{2} \zeta+m_{2} \sec \zeta \tan \zeta+1\right) \\
U_{2}(\xi, \tau)= \pm R \sqrt{\alpha+\beta^{2}}\left(-\cot \zeta+m_{1} \csc \zeta\right)-\lambda, \\
V_{2}(\xi, \tau)=\left[R^{2}\left(\alpha+\beta^{2}\right)-R^{2} \beta \sqrt{\alpha+\beta^{2}}\right]\left(\cot ^{2} \zeta-m_{2} \csc \zeta \cot \zeta+1\right)
\end{gathered}
$$

where $\zeta=R(\xi+\lambda \tau+c)-\frac{1}{2} \ln \left|\frac{A}{B}\right|, m_{1}=m_{2}= \pm 1, A^{2}+B^{2} \neq 0, A, B, R, \lambda, c$ are arbitrary constants.

Thus, we obtain following double periodic wave solutions of the WBK equations:

$$
\begin{gathered}
u_{5}(x, t)= \pm R \sqrt{\alpha+\beta^{2}}(\tan \eta+\sec \eta)-\lambda, \\
v_{5}(x, t)=\left[-R^{2}\left(\alpha+\beta^{2}\right)+R^{2} \beta \sqrt{\alpha+\beta^{2}}\right]\left(\tan ^{2} \eta+m_{2} \sec \eta \tan \eta+1\right) \\
u_{6}(x, t)= \pm R \sqrt{\alpha+\beta^{2}}\left(-\cot \eta+m_{1} \csc \eta\right)-\lambda, \\
v_{6}(x, t)=\left[R^{2}\left(\alpha+\beta^{2}\right)-R^{2} \beta \sqrt{\alpha+\beta^{2}}\right]\left(\cot ^{2} \eta-m_{2} \csc \eta \cot \eta+1\right) .
\end{gathered}
$$

where $\eta=R\left(\delta x-c_{1} \delta t+c_{2}\right)+\lambda R\left(t+c_{3}\right)+R c-\frac{1}{2} \ln \left|\frac{A}{B}\right|=R \delta x+R\left(\lambda-c_{1} \delta\right) t+R\left(c_{2}+\lambda c_{3}+c\right)-\frac{1}{2} \ln \left|\frac{A}{B}\right|, m_{1}=m_{2}= \pm 1, A^{2}+B^{2} \neq 0$, 
$A, B, R, \lambda, c$ are arbitrary constants, $\delta= \pm 1$.

\section{Conclusions}

From the theorem, it follows that the symmetry group is the product of the usual Lie point symmetry group (see [13]). Because if we take the constants in Eq.(18) and Eq.(19) as follows,

$$
c_{1}=\varepsilon C_{1}, c_{2}=\varepsilon C_{2}, c_{3}=\varepsilon C_{3}
$$

where $\varepsilon$ is an infinitesimal parameter and $C_{i}(i=1,2,3)$ are arbitrary constants. Then Eq. (18) and Eq.(19) can be written as,

$$
\begin{gathered}
u=U+\varepsilon \sigma(U), v=V+\varepsilon \sigma(V) \\
\sigma(U)=\left(\delta x-C_{1} \delta t+C_{2}\right) U_{x}+\left(t+C_{3}\right) U_{t}, \sigma(V)=\left(\delta x-C_{1} \delta t+C_{2}\right) V_{x}+\left(t+C_{3}\right) V_{t}
\end{gathered}
$$

where $\sigma(U)$ and $\sigma(V)$ is the symmetry of the WBK equations.

In fact, finishing above discussion, we can see that the equivalence transformation obtained by the direct method is more extensive and simpler than that obtained by the Lie-point group, and we can obtain even more new exact and explicit solutions if we take above solutions (20)-(21) as seed solutions.

Moreover, by applying this direct method, we can find many new solutions of other nonlinear evolution equations with variant coefficients, and this left for the future work.

\section{Acknowlegement}

This work is supported by the National Natural Science Foundation of China (41372301), Scientific Research Fund of Sichuan Provincial Education Department (14ZB0115 and 15ZB0113), Doctorial Research Fund of Southwest University of Science and Technology (15zx7138).

\section{References}

[1] M. J Ablowitz, Soliton, Nolinear Evolution Equations and Inverse Scatting, Cambridge University Press, New York, 1991.

[2] L. J. F. Broer, Approximate equations for long water waves, Applied Scientific Research 31 (1975) 377-395.

[3] Y. Chen, Q. Wang, B. Li, A generalized method and general form solutions to the Whitham-Broer-Kaup equation, Chaos, Solitons \& Fractals, 22(2004)675-682.

[4] Y. Chen, Q. Wang, Multiple Riccati equations rational expansion method and complexions solutions of the Whitham-Broer-Kaup equation, Physics Letters A, 347 (2005)215-227.

[5] Y. Chen, Q. Wang, B. Li, Elliptic equation rational expansion method and new exact travelling solutions for Whitham-Broer-Kaup equations, Chaos, Solitons \& Fractals, 26(2005)231-246.

[6] P. A. Clarkson, M. D. Kruska, New similarity solutions of the Boussinesq equation, Journal of Mathematical Physics, 30 (1989) 2201-2213.
[7] S. M. El-Sayed, D. Kaya, Exact and numerical traveling wave solutions of Whitham-Broer-Kaup equations, Applied Mathematics and Computation, 167(2005)1339-1349.

[8] E. G. Fan, H. Q. Zhang, Backlund transformation and exact solutions for Whitham-Broer-Kaup Equations in shallow water, Applied Mathematics and Mechanics, 19(1998) 667-670.

[9] X. Y. Jiao, H. Q. Zhang, An extended method and its application to Whitham-Broer-Kaup equation and twodimensional perturbed $\mathrm{KdV}$ equation, Applied Mathematics and Computation, 172(2006)664-677.

[10] D. J. Kaup, A higher-order water-wave equation and the method for solving it, Progress of Theoretical Physics 54 (1975) 396-408.

[11] S. Y. Lou, H. C. Ma, Non-Lie symmetry groups of $(2+1)$ dimensional nonlinear systems obtained from a simple direct method, Journal of Physics A: Mathematical and General, 38 (2005)129-137.

[12] H. C. Ma, A simple method to generate Lie point symmetry groups of the (3+1)-dimensional Jimbo-Miwa equation, Chinese Physics Letters, 22(2005)554-557.

[13] P. J. Olver, Applications of Lie groups to differential equations, Springer-Verlag, 1993.

[14] J. W. Shen, W. Xu, Y. F. Jin, Bifurcation method and traveling wave solution to Whitham-Broer-Kaup equation, Applied Mathematics and Computation,171(2005)677-702.

[15] Sirendaoreji, A new auxiliary equation and exact travelling wave solutions of nonlinear equations, Physics Letters A, 356 (2006)124-130.

[16] G. B. Whitham, Variational methods and applications to water waves, Proceedings of the Royal Society of London, Series A 299 (1967) 6-25.

[17] F. D. Xie, Z. Y. Yan, H. Q Zhang, Explicit and exact traveling wave solutions of Whitham-Broer-Kaup shallow water equations, Physics Letters A, 285(2001)76-80.

[18] G. Q. Xu, An elliptic equation method and its applications in nonlinear evolution equations, Chaos, Solitons \& Fractals, 29 (2006)942-947.

[19] G. Q. Xu, Z. B. Li, Exact travelling wave solutions of the Whitham-Broer-Kaup and Broer-Kaup-Kupershmidt equations, Chaos, Solitons \& Fractals, 24(2005)549-556. 
[20] T. Xu, J. Li, H. Q. Zhang,et al, New extension of the tanhfunction method and application to Whitham-Broer-Kaup shallow water model with symbolic computation, Physics Letters A, 369 (2007) 458-463.
[21] Z. Y. Yan, H. Q Zhang, New explicit solitary wave solutions and periodic wave solutions for Whitham-Broer-Kaup equation in shallow water, Physics Letters A, 285(2001) 355-362. 